\title{
A CLOSER LOOK AT THE HEALTH OF CATS SHOWING URINARY HOUSE SOILING (PERIURIA): A CASE-CONTROL STUDY
}

\author{
Ramos, D. ${ }^{1}$; Reche-Junior, A. ${ }^{1}$; Mills, D.S. ${ }^{2}$; Fragoso, P.L ${ }^{1}$.; Daniel, A.G.T. ${ }^{1}$; \\ Freitas, M.F. ${ }^{1}$; Cortopassi, S. ${ }^{3} ;$ Patricio, $\mathrm{G}^{3}$.
}

1 - Department of Medical Clinics, Faculty of Veterinary Medicine and Animal Science, University of

São Paulo, Brazil.

18

2 - Animal Behaviour, Cognition and Welfare Group, School of Life Sciences, University of Lincoln,

UK; Lincoln, LN6 7DL 


\section{Abstract}

Objective: Perform a case-control medical evaluation of cats from multi-cat households presenting with inappropriate latrining and spraying behavior.

Methods: Owners of 18 spraying and 23 latrining cats with normal control subjects available from the same households were recruited for a case-control study. Otherwise overtly healthy dyads were brought together to the veterinary hospital of the University of São Paulo, for a medical work-up (i.e. physical examination, complete blood count, biochemical profile, urinalysis and urine culture, abdominal ultrasound of the urinary system and in females, where possible, cystoscopy).

Results: Medical problems were identified with similar frequency in the "sprayers" (38.9\%) "latriners" (39.1\%) and controls of the latrining group (26.1\%) but not the spraying group $(5.5 \%)$. The difference between cases and controls from spraying households was significant. Common potential health related changes include renal insufficiency, cystitis, bladder lithiasis. Renal calculi, higher creatinine levels (within normal reference range) and "glomerulations" (detected during cystoscopy) were also found in the remaining "sprayers" and "latriners" who were considered healthy. Post-cystoscopy a new form of periuria occurred in two cats (one "sprayer" and one "latriner").

Conclusions and Relevance: These results indicate that spraying or latrining behaviour in the home, as well as living with a cat who is not using the litter box as a latrine, are all associated with a higher level of urinary tract abnormalities, but not living with a cat who is spraying. The findings also suggest that both forms of periuria might be associated with interstitial cystitis. We therefore conclude that all cats with periuria need to be carefully evaluated medically and that treatment of latrine related problems should consider all cats in the house, whereas spraying may be more focused on the individual displaying the problem.

Key-words: marking, spraying, toileting, latrining, disease, feline, cystoscopy 


\section{INTRODUCTION}

In the clinical context, spraying for marking purposes by domestic cats has long been distinguished from inappropriate urinary latrine related behavior (latrining) $(1,2,3,4,5,6,7,8)$. Spraying commonly involves urine deposited on vertical surfaces (or on significant horizontal spots) with the cat typically in a standing posture $(2,4,5)$. "Sprayer" cats generally keep use of the appropriate latrine for both urination and defecation $(2,4,5)$. By contrast, inappropriate latrining is mostly characterized by large amounts of urine, usually on horizontal surfaces with the cat in a squatting posture $(2,4,5)$. "Latriner" cats may give up using the latrine and so both urine and faeces may be found in inappropriate locations $(2,4,5)$.

Successful management of either condition depends on the identification of medical factors which might be contributing to the problem $(1,2,3,4,5,6,7,8)$. The evidence for the significance of medical problems in these cases is inconsistent $(9,10)$, and largely based on simple case reports and comparisons, with inadequate controls for things like differences in management that might exist (11). Nonetheless, the overall recommendation of adequate medical screening in order to eliminate potential medical factors contributing to the problem, remains sound advice $(1,2,3,4,5,6,7,8)$. Some suggest that the urination behaviour of cats with a urinary problem does not resemble urine marking, and urinalysis does not provide relevant diagnostic information, at least in cases of urine spraying (12), but urinalysis may be of value in cases of latrine related problems (12). It is also worth noting that in many cases of periuria (problematic deposition of urine in the home regardless of cause), the medical assessment is often quite superficial, frequently based on only a brief physical exam and possibly urinalysis (12). A more complete evaluation of the urinary system (e.g. via ultrasound or cystoscopy) may reveal further abnormalities (13). Indeed, the frequency and extent to which medical evaluations are made in these cases is not known. Thus it is not possible to say with any confidence, what problems are present in these cases or what their relevance might be. Current practice focuses on the behavioural history, with the precise role of urinary system abnormalities evaluated to a variable extent for their relevance on a case by case basis.

Veterinary behavioural guidelines not only propose diagnostic procedures but also treatment

101 protocols, for both inappropriate latrining behaviour and spraying. Although these are generally clear and reasonably easy to apply, complete eradication of the problem using only management and environmental change is often unsuccessful (14). There is clearly a proportion of refractory cases in 
which typical management regimes do not work. This may include those with untreated medical complications, and so it is important to improve our understanding of the potential relationship between and role of urinary tract abnormalities in these cases. of the association between urinary tract disease and periuria by performing an in-depth medical evaluation of a series of cats from multi-cat households presenting with inappropriate latrining and

110 spraying behaviour alongside control subjects drawn from the same household in order to control for

111 management confounds between subjects (15).

\section{MATERIAL AND METHODS}

\subsection{Recruitment and Selection}

the same multi-cat households ( $n=3-9$ cats per house) was promoted in several ways: via a poster at the veterinary college at the University of São Paulo (FMVZ/USP) and several veterinary clinics in Sao Paulo and neighboring cities, emails to a list of students of the veterinary college, postings on cat breeder websites and Internet communities related to pet cats.

Cats reported by their owners to manifest periuria were classified into spraying or latrining groups (together with their respective controls without periuria) on the basis of a careful behaviour analysis made by a veterinary behaviourist considering cat posture and behaviour repertoire when depositing urine outside the litterbox, location, target and amount of urine deposited outside the litterbox, litterbox use, etc. Households where different cats exhibited urine spraying and latrine related behaviour in the home were not eligible for inclusion. This means that in selected spraying households there was no latrining behaviour in the home by any of the cats; similarly, in the selected latrining households a spraying problem was absent. receive any treatment for their problem behaviour. 
Twenty-one urine spraying "case-control" dyads were initially recruited, but in the case of three

136 dyads examinations could not be concluded for various reasons (e.g. cat became sick) and therefore

137 they were excluded from further involvement in the research. The remaining 18 "case-control" dyads

138 were then subjected to medical examinations involving a complete blood count, biochemical profile,

139 urine exam and urine culture and ultrasonic evaluation of the urinary system at the university veterinary

140 hospital (HOVET-USP).

141 Among the 18 "case" cats, there were 3 females and 15 males; 17 mixed-breeds and one

142 Persian, with average age 6.27 years $(\mathrm{SD}=2.44)$. "Control" cats were 6 females and 12 males; 17

143 mixed-breeds and one Siamese, with average age 6.43 years old $(\mathrm{SD}=4.48)$. On average, there

144 were 6.09 cats per household (range: 3-8 cats). Four of the 18 "case-control" dyads came from

145 two households; there were therefore 16 different households in this Group. Spraying

146 households were all houses (as opposed to flats) and half of them offered free outside access to the 147 cats.

$148 \quad$ 2.1.2 Latrining Group

149 Twenty-nine inappropriate latrining "case-control" dyads were initially recruited but for six

150 dyads the examinations could not be concluded (e.g. owner did not fast in the cat or did not prevent the

151 litterbox use and so cat did not have sufficient urine for the exams). The remaining 23 "case-control"

152 dyads were then submitted to similar clinical examinations at the university veterinary hospital

153 (HOVET-USP).

154 Of the selected 23 "case-control" dyads, 18 "case" cats were females and 5 males; 13 155 mixed-breeds, 4 Persians, 4 Maine Coons and 2 Ragdolls. Their average age was 4.46 years 156 ( $\mathrm{SD}=2.19)$. Among the "control" cats, there were 13 females and 10 males; 16 of mixed-breed, 4 157 Maine Coons and 3 Persian. Their average age was 5.21 years old ( $\mathrm{SD}=3.35)$. Households in 158 this group had on average 4.6 cats (range: 3-8 cats). Four of the 23 "case-control" dyads came 159 from two households, and so there were 21 different households in the group. Toileting 160 households were evenly split between houses and flats and none of the toileting households offered free 161 outside access to the cats. 
All procedures involved in the medical examination were conducted on both "case" and "control" cats from the household on the same morning, with cats having fasted for between 8 and 12 hours. The collected materials were processed according to standards and techniques established by the Laboratory of Clinical Pathology (HOVET-USP). Ultrasounds were performed in the same room, by a specialist in veterinary ultrasonography; a second veterinary feline specialist carried out all other clinical examinations.

In the 2 hours immediately preceding the exams, owners were instructed to encourage water intake, and to prevent the use of the litterbox in an attempt to promote bladder filling, necessary for the collection of urine, which was done by cystocentesis guided by ultrasound. Blood samples had to be sufficient for at least a complete blood count and biochemistry profile, otherwise the dyad was excluded.

Any abnormality of the lower urinary tract (e.g. bladder urolithiasis, cystitis) or alterations indicative of either systemic disease or organ dysfunction was considered a medical abnormality; the dyad did not then progress further in order to avoid undue stress, following the establishment of a medical problem of concern. However, alterations of the upper urinary tract which, did not appear to interfere with functioning of the urinary system (e.g. renal urolithiasis accompanied by normal

180 urinalysis and renal profile) although considered potentially problematic, did not result in exclusion of 181 the dyad from further analysis.

\subsection{Cystoscopy}

184 Second visits to the veterinary hospital were planned for cystoscopies of suitable female cats, subject to owner informed consent, including explanation of the need for a general anaesthetic.

186 Cystoscopies were carried out on selected female "case" cats only (i.e. those exhibiting periuria without medical abnormality of concern in the preliminary exams). This cystoscopy allowed us to investigate abnormalities that may have passed unnoticed in the previous exams (e.g. interstitial cystitis) (13).

190 Group and 10 from the Latrining Group 2) included female "case" cats, suitable for cystoscopic examination and 8 owners elected for cystoscopy. 
194 were pretreated with acepromazine and meperidine $(0.05 \mathrm{mg} / \mathrm{kg}$ and $4 \mathrm{mg} / \mathrm{kg}$, respectively, IM).

195 Anesthesia was then induced with propofol $(5-6 \mathrm{mg} / \mathrm{kg}$, IV) and maintained with isoflurane diluted in

$196100 \%$ oxygen at concentrations necessary to maintain the third level of the third stage of Guedel's

197 classification of depth of anesthesia (16). In the case of three females which appeared to be aggressive,

198 ketamine (2 mg/kg IV) was added to the premedication, which allowed us to safely handle them whilst

199 promoting induction with propofol. In the last four procedures, in order to achieve a better penetration

200 of the endoscope via a deeper urethral relaxation, butorphanol $(0.4 \mathrm{mg} / \mathrm{kg} \mathrm{IV})$ was added to the 201 protocol.

\subsection{Statistical Analysis}

A Shapiro-Wilks normality test was first performed for each of the medical variables in both groups ("cases" and "controls" were first tested separately). In the event of a normal distribution for both "cases" and "controls", Student paired t-tests were used for pairwise comparisons, with Wilcoxon signed-rank tests used in the case of non-normally distributed data.

The prevalence of medical alterations in "case" versus "control" cats in both spraying and inappropriate latrining dyads was compared by either Fisher exact test or Pearson $\mathrm{Chi}^{2}$ test. Similarly,

210 comparisons of case subjects between groups were also assessed using Pearson $\mathrm{Chi}^{2}$ test. Statistical

211 analysis was performed using SAS software (9.2 version) with a probability level of 0.05 used to assess 212 significance.

\section{RESULTS}

\subsection{Spraying dyads}

218 (38.9\%) demonstrated medical alterations; but this was found in only one control cat (5.5\%). 219 Significantly more "case" than "control" cats (Fisher exact test, $\mathrm{p}=0.041$ ) therefore had at least one of 220 the following medical conditions: renal insufficiency (2), idiopathic cystitis (2), diabetes insipidus (1), 221 diabetes mellitus (1), ectopic testes (1). The only control cat with a medical alteration presented with 222 bladder lithiasis. 
224 screen, although some medical complications which did not obviously impair either urinary or other 225 system functioning were identified. In 4 "case" and 1 "control" cat, renal lithiasis was identified (these 226 measured about $0.3 \mathrm{~cm}$ and in all cases were located in the renal pelvis); a renal cyst was found in a 227 fifth "case" cat. One "case "and one "control" showed renal diverticulum calcification. This meant that 22812 out of the initial 18 recruited "sprayers" (i.e. 66.7\%) and 3 out of 18 (i.e. 16.7\%) controls had 229 identifiable deviations from a perfect state of the urinary tract. One of the remaining "case" cats had 230 glycosuria, but blood levels of glucose as well as frutosamine were within normal reference range. 231 Leucocytes count was high (i.e. 19,900 cells $/ \mathrm{mm}^{3}$ ) and further urinalysis did not reveal glycosuria 232 suggesting the condition was not due to diabetes mellitus.

233 Blood leucocyte counts of "cases" and "controls" (n=11) did not differ significantly (median $234+$ +- IQR: "case" cats $=13,700+/-3,800$ cells $/ \mathrm{mm}^{3}$, controls $=10,700+/-13,200$ cells $/ \mathrm{mm}^{3} ; \mathrm{p}=$ 235 0.6377). However, serum creatinine levels were significantly higher for sprayers in comparison with 236 their "controls" (mean+/-SD: "case" cats $=1.53+/-0.31 \mathrm{mg} / \mathrm{dL}$ controls $=1.19+/-0.23 \mathrm{mg} / \mathrm{dL} \mathrm{p}=0.005)$. 237 With regard to blood urea, the difference between "cases" and "controls" approached significance $($ mean+/-SD: "case" cats $=62.68+/-6.13 \mathrm{mg} / \mathrm{dL}$ controls $=56.35+/-6.41 \mathrm{mg} / \mathrm{dL} \mathrm{p}=0.0597)$. submucosal petechiae ("glomerulations") (Figure 1), oedema and mild bladder haemorrhage, indicative of interstitial cystitis.

Follow-up: over the days following cystoscopy, the cat with petechiae showed hematuria, 243 polakiuria and apparently painful urination. A latrine related problem arose with the cat depositing 244 urine containing blood in the bathroom sink (such a behaviour had never been observed before). This 245 lasted a couple of weeks and the owner felt that the problem had been exacerbated by the cystoscopic examination. The cat was successfully treated with amitriptyline $(1 \mathrm{mg} / \mathrm{kg}$, BID) from the first day after 247 cystoscopy for 60 days; the cat returned to using the litterbox for latrine related urination with the owner reporting only rare occasions of urine spraying. Such improvement was sustained for at least 6 months when the last contact with the owner occurred.

251 Figure 1. Cystoscopic examination of a female "sprayer" cat. White arrows indicate petechiae (A normal size; B $-1.5 \mathrm{X}$ zoom). 


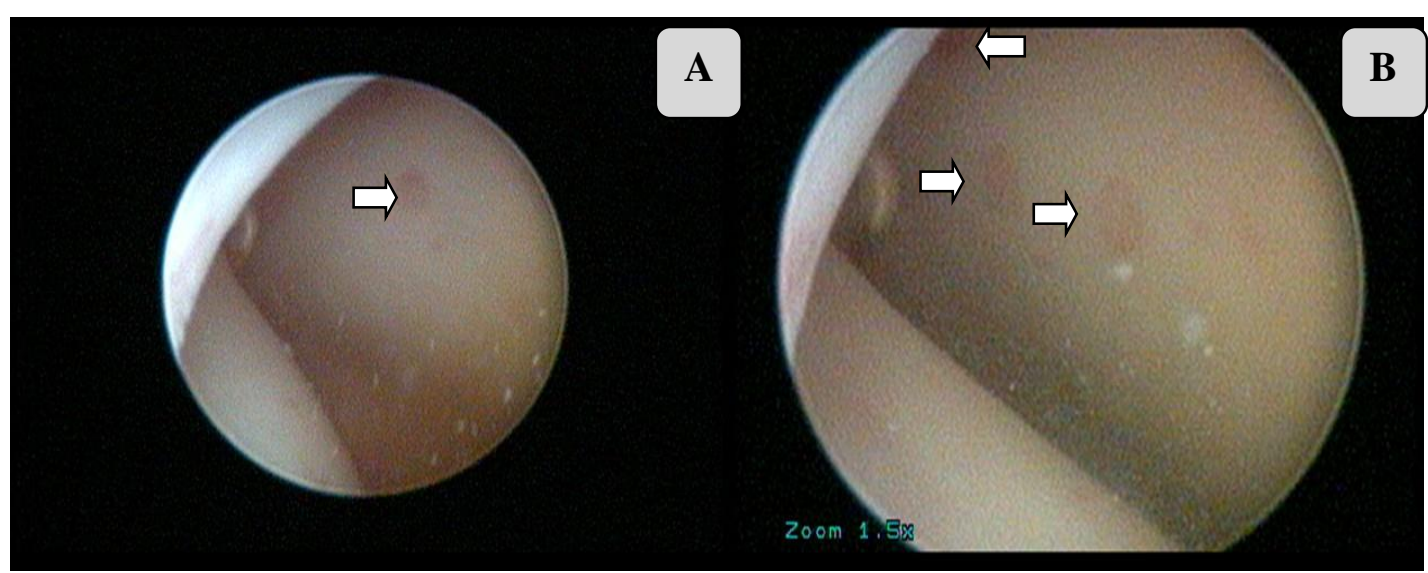

\subsection{Latrining dyads}

261 behaviour, 9 (39.1\%) demonstrated an overt medical concern; this was found in 6 control cats (26.1\%).

262 The difference was not significant between "case" and "control" cats (Pearson Chi ${ }^{2}$ test, $\mathrm{p}=0.365$ ).

263 Medical problems found among cases were: renal insufficiency (3), leucocytosis (2), leucocytosis plus

264 bladder plug (1), bladder lithiasis (1), bladder plug (1), hepatic disease (1). "Control" cats had 265 leukaemia (1), bladder diverticulum (1), renal insufficiency (1), bladder lithiasis (1), abdominal liquid 266 (1), hepatic disease plus urinary infection (1).

267 Of the 12 remaining dyads considered healthy on the basis of the initial health screen, there 268 were a range of other conditions identified which were not considered to obviously impair either 269 urinary or other organic system functioning. Four "case" and 4 "control" cats had one of the following 270 alterations in the ultra-sound image of their kidneys: diverticulum calcification, heterogenous texture, 271 decrease cortico-medullary definition, retraction and/or thinning of cortical area leading to loss of 272 kidney definition. This meant that in total 13 out of the initial 23 recruited "latriners" (56.5\%) had 273 abnormalities and 10 out of 23 controls (43.5\%).

274 One "control" cat had glycosuria, but blood levels of glucose and frutosamine were within 275 normal reference ranges and leucocytes count was high (i.e. 30,100 cells $/ \mathrm{mm}^{3}$ ). Later further urinalysis 276 did not reveal glycosuria suggesting that the cat did not have diabetes mellitus. 

SD: "case" cats $=10,641.67+/-2,191.91$ cells $/ \mathrm{mm}^{3}$, controls $=13,308.33+/-7,206.87$ cells $/ \mathrm{mm}^{3} ; \mathrm{p}=$

279 0.229). Serum creatinine as well as urea levels were also not significantly different when "latriners" 280 were compared with their controls (mean+/-SD, creatinine: "case" cats $=1.38+/-0.15 \mathrm{mg} / \mathrm{dL}$, controls = $2811.40+/-0.26 \mathrm{mg} / \mathrm{dL} \mathrm{p}=0.848$; urea: "case" cats $=60.28+/-15.58 \mathrm{mg} / \mathrm{dL}$ controls $=60.13+/-12.66 \mathrm{mg} / \mathrm{dL}$ $\mathrm{p}=0.968)$.

Cystoscopy was attempted on 6 of the 10 potentially qualifying female cats, but complete

284 bladder visualization was possible in only 3 of them; in the other 3 cats, urethra passage was very 285 difficult and given the risk of injury, the procedure was abandoned. The bladder was healthy in 2 cats, 286 but in the third there were submucosal petechiae ("glomerulations"), ulcerations and haemorrhage 287 (Figure 2) indicative of interstitial cystitis.

Follow-up: on the days following cystoscopy the cat diagnosed with interstitial cystitis showed 289 hematuria, polakiuria and painful urination (including high pitch vocalization before entering the litter 290 box). Urine spraying, which had never been observed in this cat before as well as a digging behaviour 291 at the front door of the house (followed by urination just there) arose during this time. This cat was treated with amitriptyline ( $1 \mathrm{mg} / \mathrm{kg}$, BID) from the first day after cystoscopy and for 90 days. Within a

293 couple of weeks, the cat returned to its previous pattern of eliminating large amounts of urine on 294 horizontal surfaces in the home without signs of pain. The owner reported, however, a decrease in 295 frequency of inappropriate urination. The problem was still present after 6 months despite inclusion of behaviour therapy to augment treatment. 


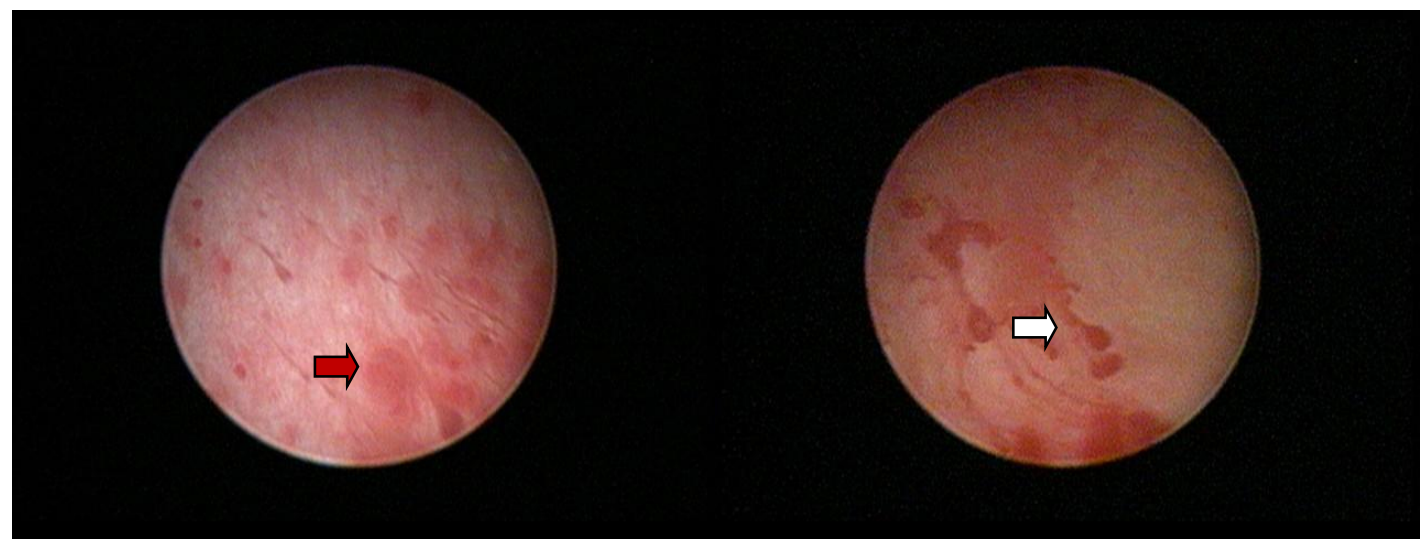

\subsection{Comparison between "Sprayers" and "Latriners"}

Medical conditions were detected with similar frequency in both apparently healthy "sprayers" and "latriners" (Pearson Chi test, $\mathrm{p}=0.639)$.

\section{DISCUSSION}

Medical conditions were common in both overtly healthy "sprayers" and "latriners", thus, despite

310 recommendations to focus on ruling out medical problems in cases of inappropriate latrining only (12),

311 our findings from the case-control study emphasize that a medical work-up should be undertaken in

312 any case of periuria as previously suggested (10). This finding is supported by two cases who went on 313 to develop periuria one in the form of spraying the other in the form of latrining, following cystoscopy.

314 The prevalence of medical conditions among our selected sprayers is more in agreement with the 315 findings of Frank et al (10), Barcelos et al (11) and Tynes et al (12) than the report of Landsberg and 316 Wilson (17). The differences could be due to which medical conditions are considered as possibly 317 interfering with the act of urine expulsion. In the study reported here, any alteration of the upper 318 urinary system potentially compromising urinary function, any abnormality of the lower urinary tract or 319 altered functioning of the organism in general that was considered as possibly linked to periuria led to a 320 medical concern for the cat; by contrast, other studies have only considered lower urinary tract 321 disorders or the result of a single urinalysis as exclusionary criteria $(12,17)$. In the case of "latriners", 322 the percentage of cats presenting a medical condition (i.e. $32.1 \%$ ) is consistent with previous research 323 which has indicated a similar lifetime prevalence of urinary tract disease $(9,11,18)$.

324 Physical alterations in periuric cats are typically described in relation to lower urinary tract 
tract were seen in both "sprayers" and "latriners" subject to more intense medical evaluation. The significance of this remains unclear. Investigations on the link between renal alterations as early renal 328 disease and different types of urinary act, particularly when urinary function does not seem to be 329 diminished are lacking. Likewise, the potential for renal formations (e.g. calculi within the kidney) to 330 cause pain and as a consequence change urination behaviour has been completely overlooked in the 331 scientific literature. Thus, if such conditions act either as causes or contributors to inappropriate 332 urination (particularly in the spraying cases) and have not been detected due to medical exams being 333 focused on the lower urinary tract, this might explain, at least in part, the refractoriness of some cases: 334 since part of the problem has not been recognised and managed. Since the veterinary goal is to provide 335 complete care for the feline patient with a urinary behaviour problem $(4,5,8)$, a detailed medical 336 examination including imaging evaluation of the upper urinary tract should be encouraged, especially 337 in refractory cases. Therapeutic intervention for some conditions (e.g. evidence of CKD-stage 1, renal 338 calculi, renal cyst) may not involve much more than monitoring the condition, since the significance of 339 these features to wider kidney disease progression is still unclear (19); a relapse in periuria correlated 340 with medical progression, or an improvement in association with medical treatment, may help to 341 establish a link between these conditions, and help to explain why a refractory case has not achieved 342 success despite the appropriate behaviour management.

343 In spraying households medical conditions were more common in "sprayers" than in "controls" 344 whereas in latrining households "latriners" and "controls" were equally affected by medical problems. 345 A 21.4\% prevalence of medical problems among "control" cats in the latrining group was unexpected; 346 a previous report of 100 apparently clinically healthy cats has indicated that only $6 \%$ had medical 347 problems (e.g. hyperthyroidism, urinary infection)*. ${ }^{1}$ The current report is the first time medical 348 problems have been identified with such high frequency in cats sharing the same environment as those 349 with a latrine problem. Nonetheless, the relatively high prevalence of abnormalities in control cats from 350 households with a latrine cat, is similar to the general prevalence reported by Tynes et al. (12) and 351 lifetime owner-reported history prevalence or urinary disease reported recently by Barcelos et al. (11). 352 One possible explanation for such a result lies in environmental physical and/or social characteristics 353 (or even ownership style) which may predispose all household cats to diseases in latrining households. 354 For example, Barcelos et al. (11) recently reported that cats with a latrine problem were less likely to be

\footnotetext{
${ }^{I}$ *Garcia JL, Bruyette DS. American College of Veterinary Internal Medicine Forum poster session. 1998.
} 
described as having a heavy dependence on their owner compared to either spraying cats or nonperiuric controls.

Spraying households had a higher average number of resident cats than latrining houses. Although this had not reached significance we can hypothesized that cats from spraying houses were more likely to be involved in conflict behaviours and therefore more prone to manifest spraying but not necessarily to develop more diseases. It should also be noted that cats in latrining houses did not have outside access whereas half of spraying households offered free outside access to the cats, and this has been identified as a risk factor for the condition (11). We hypothesise that a lack of access to diverse external latrines might reduce urinary frequency and thus encourage overdistension of the bladder, predisposing subjects to cystitis. Depending upon combinations of external factors (e.g. decrease in water availability, provision of a low quality diet) and individual predispositions, medical problems interfering with the urinary system may also arise, potentially leading to periuria in some subjects. In other cases, non-urinary illness may develop and likely result in other medical signs which may not be perceived by the owner of multiple-cats, at least in its early stages. Thus, we suggest that there may be several ways in which the care needed for optimal bladder health may not be provided by the owners of participant cats in the latrining group. Whether this is a problem of the households in this study only, or if it represents a wider characteristic in cases of latrining cats remains to be explored, although the recent results of Barcelos et al. (11) suggest it may be a much wider problem. In the interests of caution to safeguard cat health and welfare, we therefore suggest clinicians make a broader enquiry into the overall environmental management and general feline urinary health care of households affected by a latrining problem. This should emphasise the importance of multiple litter tray sites in all homes, with indoor only cats (regardless of the number of cats in the household), but perhaps for a slightly different reason than is commonly described in behaviour texts $(1,2,4,5,6,8)$; i.e. to maintain bladder health rather than meet the desire of cats not to share a latrine with others. Although issues such as unacceptable cleanliness of the litterbox, inappropriate distribution of the litterboxes etc may play a

381 Barcelos et al. (11), although lack of access to outdoor latrines was. Perhaps too much emphasis has 382 been put on hygiene and odour related issues, when it may be more appropriate to focus on choice and opportunity for cats. 

of a more multimodal veterinary medical approach to behaviour cases $(20,21,22,23,24,25)$. However, as professionals it is important for veterinarians to offer expert advice which balances the risks with the benefits of any proposed procedure. We do not recommend cystoscopy for all female cases of

388 inappropriate urination. There are risks from anaesthesia, possible complications (including those 389 illustrated here) and cost factors to consider, alongside a recognition that full inspection of the bladder 390 is not possible in all cases (25). It should also be recognised as a highly specialised procedure $(26,27)$. 391 However we do suggest that cystoscopy may be valuable in refractory, atypical and more obscure cases. Before cystoscopy, owners must be made aware of the procedure itself as well as the risk of temporary worsening of the problem or a new form of periuria, as appeared to occur in two cases in this study. home, we suggest that attention needs to be paid to the provision of sufficient latrine opportunities as a first line of enquiry. Further work is required to establish the nature of the causal link between urinary tract problems and periuria, and this will likely be accelerated by greater co-operation between internal

\section{CONCLUSIONS}

In conclusion, our findings emphasise the importance of considering not just bladder health but

\section{5. REFERENCES}

413 1. Landsberg G, Hunthausen W, Ackerman L. Feline Housesoiling. In: Landsberg G,

414 Hunthausen W, Ackerman L. Handbook of behavior problems of the dog and cat. Edinburgh:

415 Elsevier Saunders; 1997. p. 365-384. 
417 canine and feline behavioural medicine. Gloucester: British Small Animal Association; 2002. p. $418 \quad 97-108$.

419 3. Beaver B V. Feline Behaviour: a guide for veterinarians. 2. ed. New York: Elsevier Inc., 4202005.

421 4. Neilson J C. Feline house soiling: elimination and marking behaviors. Clin Tech Small An P. 422 2004a; 19 (4): 216-224.

423 5. Neilson J C. Thinking outside the box: feline elimination. J Feline Med Surg. 2004b; 6: 5-11.

424 6. Bowen J S, Heath S. Feline housesoiling and marking problems. In: Bowen J S, Heath S. 425 Behavior problems in small animals: practical advice for the veterinary team. Philadelphia: 426 Elsevier Saunders; 2005. p. 185-203.

427 7. Horwitz D F, Neilson J C. Housesoiling: feline. In: Horwitz D F, Neilson J C. Canine and 428 feline behaviour. Blackwell's five minutes veterinary consult - clinical companion. Iowa: 429 Blackwell Publishing; 2007. p. 329-336.

430 8. Herron M E. Advances in understanding and treatment of feline inappropriate elimination. 431 Top Companion Anim M. 2010; 25 (4): 195-202.

432 9. Horwitz D F. Behavioral and environmental factors associated with elimination behavior 433 problems in cats: a retrospective study. Appl Anim Behav Sci. 1997; 52: 129-137.

434 10. Frank D F, Erb H N, Houpt K A. Urine spraying in cats: presence of concurrent disease and 435 effects of a pheromone treatment. Appl Anim Behav Sci. 1999; 61: 263-272.

436 11. Barcelos A M, Mcpeake K, Affenzeller N, Mills D S. Common risk factors for urinary house 437 soiling (periuria) in cats and its differentiation: the sensitivity and specificity of common 438 diagnostic signs. FVETS. 2018; 5(108): 1-12.

439 12. Tynes V V, Hart B L, Pryor P A, Bain M J, Messan L L. Evaluation of the role of lower 440 urinary tract disease in cats with urine-marking behavior. JAVMA. 2003; 223 (4): 457-461.

441 13. CHEW, D. J.; BUfFINGTON, C. A. T. Idiopathic / intersticial cystitis: diagnosis and 442 management. Proceedings of the Annual Seminars of the Companion Animal Society of the NZVA, 443 Proceedings of the Annual Seminar. FCE Publication; 2004, No. 235.

444 14. Mills D S, Redgate S E, Landsberg G M. A meta-analysis of studies of treatments for feline urine spraying. PLoS One. 2011; 6(4): e18448. 

University Press, 1982.

448 16. Guedel A E. Inhalation anesthesia: a fundamental guide. Scotland: Macmillan, 1937.

449 17. Landsberg G M, Wilson A L. Effects of clomipramine on cats presented for urine marking. J 450 Am Anim Hosp Assoc. 2005; 41 (1): 3-11.

451 18. Olm D D, Houpt K A. Feline house-soiling problems. Appl Anim Behav Sci. 1988; 20: 335452345.

453 19. Ross S J, Osborne C A, Leckcharoensuk C, Koehler L A, Polzin D J. A case-control of the 454 effects of nephrolithiasis in cats with chronic kidney disease. JAVMA. 2007; 230 (12): 18544551859.

456 20. Chew D J, Buffington C A T, Kendall M S, DiBartola S P, Woodworth B E. Amitriptyline 457 treatment for severe recurrent idiopathic cystitis in cats. JAVMA. 1998; 213 (9): 1282-1286.

458 21. Westropp J L, Buffington C A T. Feline idiopathic cystitis: current understanding of 459 pathophysiology and management. Vet Clin N Am-Small. 2004; 34: 1043-1055.

460 22. Gunn-More D A, Cameron M E. A pilot study using synthetic feline facial pheromone for 461 the management of feline idiopathic cystitis. J Feline Med Surg. 2004; 6 (3): 133-138.

462 23. Gunn-More D A, Shenoy C M. Oral glucosamine and the management of feline idiopathic 463 cystitis. J Feline Med Surg. 2006; 6 (4): 219-225.

464 24. Buffington C A T, Westropp J L, Chew D J, Bolus R R. Clinical evaluation of multimodal 465 environmental modification (MEMO) in the management of cats with idiopathic cystitis. J 466 Feline Med Surg. 2006; 8 (4): 261-268.

467 25. Seawright A, Casey R, Kiddie J, Murray J, Gruffydd-Jones T, Harvey A, Hibbert A. A case 468 of recurrent feline idiopathic cystitis: the control of clinical signs with behavior therapy. J Vet 469 Behav. 2008; 3 (1): 32-38.

470 26. Messer J S, Chew D J, McLoughlin M A. Cystoscopy: techniques and clinical applications. 471 Clin Tech Small An P. 2005; 20 (1): 5264.

472 27. McCarthy T. C. Cystoscopy. In: McCarthy T. C. Veterinary endoscopy for the small animal 473 practioner. Missuri: Elsevier Saunders; 2005. p. 49-135. 